\title{
272. Ergebnisse einer prospektiv randomisierten Studie beim operablen Mammacarcinom
}

\author{
R. Kolb, G. Reiner, R. Jakesz, K. Moser und M. Schemper \\ I. Chirurgische Universitätsklinik Wien, Alserzstraße 4, A-1090 Wien
}

\section{Results of a Randomized Prospective Trial in Operable Breast Cancer}

Summary. Surgical treatment, partial resection (R) versus modified radical mastectomy (M) and $M$ versus the classical Halsted procedure (RM) as well as adjuvant treatment forms, chemotherapy and chemoimmunotherapy versus an untreated control group were all evaluated in 241 patients, with a median observation time of 30 months. Whereas $M$ showed significantly better results than $R$, no difference could be observed when comparing $\mathrm{M}$ and $\mathrm{RM}$. The disease-free interval was significantly reduced in the control group as compared to patients receiving chemotherapy. No benefit could be detected in patients with immunotherapy (azimexon).

Key words: Breast cancer - Prospective randomized trial.

Zusammenfassumg. Im multifaktoriellen Versuchsansatz wurden operatives Vorgehen, Resektion (R) versus modifiziert radikale Mastektomie (M) und $M$ versus klassisch radikaler Mastektomie (RM), und Fragen der adjuvanten Therapie, Chemo- und Chemoimmunotherapie gegenüber einer Kontrollgruppe, an 241 Patientinnen nach dem rezidivfreien Überleben - 30 Monate mediane Beobachtungszeit - beurteilt. R schnitt gegenüber $M$ signifikant schlechter ab. Dagegen zeigte $M$ gleich gute Ergebnisse wie RM. Fälle mit Chemotherapie waren gegenüber der Kontrollgruppe signifikant besser dran. Keine Verbesserung brachte die Immunotherapie mit Azimexon.

Schliusselwörter: Mammacarcinom - Prospektiv randomisierte Studie.

\section{Probleme und Komplikationen der Mammarekonstruktion nach Mastektomie}

\author{
H. Mandl, G. Freilinger, J. Holle und M. Frey
}

Abteilung für Plastische und Wiederherstellungschirurgie der II. Chirurgischen Universitätsklinik Wien, Spitalgasse 23, A-1090 Wien

\section{Problems and Complications in Breast Reconstruction After Mastectomy}

Summary. Reconstruction of the female breast after mastectomy clearly improves the psychic situation of the woman. Several methods are available for breast reconstruction: local or myocutaneous flap techniques after radical mastectomy, subcutaneous or subpectoral implantation of prosthesis or coriumplasty after subcutaneous mastectomy. Of 83 females who underwent breast reconstruction by one of these methods between 1976 and 1982, 62 were checked up in 1982. The problems and complications of breast reconstruction were critically analysed.

Key words: Breast reconstruction.

Zusammenfassung. Die Wiederherstellung der weiblichen Brustform nach Brustamputation verbessert deutlich die psychische Situation der betroffenen Frauen. Für die Rekonstruktion stehen verschiedene Methoden zur Verfügung: lokale Hautlappen oder myocutane Lappenplastiken nach querer Mastektomie, subcutane oder subpectorale Prothesenimplantation oder Coriumplastik nach subcutaner Mastektomie. 62 von 83 Frauen, bei denen 1976-1982 nach einer dieser Methoden eine Brustrekonstruktion durchgeführt worden war, wurden 1982 nachuntersucht. Die Probleme und Komplikationen der Brustrekonstruktion werden kritisch analysiert.

Schlüsselwörter: Brustrekonstruktion - Mammarekonstruktion. 